\title{
Electronic response and bandstructure modulation of carbon nanotubes in a transverse electrical field
}

\author{
Yan Li, Slava V. Rotkin, Umberto Ravaioli \\ Beckman Institute for Advanced Science and Technology \\ University of Illinois at Urbana-Champaign \\ Urbana, IL 61801 USA
}

\begin{abstract}
The electronic properties of carbon nanotubes (NTs) in a uniform transverse field are investigated within a single orbital tight-binding (TB) model. For doped nanotubes, the dielectric function is found to depend not only on symmetry of the tube, but also on radius and Fermi level position. Bandgap opening/closing is predicted for zigzag tubes, while it is found that armchair tubes always remain metallic, which is explained by the symmetry in their configuration. The bandstructures for both types are considerably modified when the field strength is large enough to mix neighboring subbands.
\end{abstract}

Keyword: theoretical, nanotubes, polarizability, dielectric function, electronic bandstructure, bandgap engineering

\section{Introduction}

The response of nanotubes to external electric fields is of interest both for transport devices[1] and for nano-electromechanical systems [2, 3]. When the transverse field strength is large enough to couple neighboring subbands, it is not appropriate to use the conventional 1D approximation, which assumes a uniform charge and potential distribution along the NT circumference. In this article, we address the issue of how the NT material properties are modified when the electronic potential along the circumference is no longer uniform. We use a single $\pi$ orbital tight-binding model in a self-consistent way to account for screening effect caused by the non-uniform charge distribution along the NT circumference.

To our knowledge, this is the first study that considers a wide range of applied transverse electric field conditions. Within the limit of relatively weak electric fields, we obtain different behaviors for semiconducting and metallic nanotubes, either intrinsic or doped. The weak field condition can be expressed as $\mathcal{E} \ll t a /\left(e R^{2}\right)(\sim 0.1 \mathrm{~V} / \AA$ for $R=8 \AA)$, where $t=2.5 \mathrm{eV}$ and $a=2.49 \AA$ are the hopping integral and lattice constant of two-dimensional graphite respectively, as defined for example in the book [4. One may safely use a rigid bond approximation in this field limit, without severely perturbing the original bandstructure. We also investigate the regime of relatively strong fields, $t a /\left(e R^{2}\right)<\mathcal{E} \ll t /(e a)$, where bandstructure modifications need to be considered and subband mixing cannot be neglected, while a TB approach can still be used. Also for this regime, qualitatively different behaviors of semiconducting and metallic NTs are found.

The paper is organized as follows. In Sec.2 we calculate the transverse polarizability for nanotubes of various symmetry, considering a linear approximation and no phonon contributions. The dependence of the polarizability on tube radius, symmetry and Fermi level position are discussed. The role of the Fermi level is not related to intra-band free carrier transitions, but rather to inter-band transitions between neighboring conduction/valence bands. We then exam the effect of a uniform transverse transverse field on the bandstructure of nanotubes in Sec.3. Because of the symmetry of the nanotube, the subbands of the tube are mixed according to certain selection rules and the bandstructure and bandgap are correspondingly modified. Several interesting phenomena are predicted to happen in the transverse field: bandgap opening/closing, energy subband flattening and over-bending, lifting of subband degeneracy, and generation of multiple valleys. These effects can be used to modulate the bandgap, effective mass and carrier densities of NTs and to enhance the density of states(DOS) at the Fermi level. For example, it has been suggested that opening of energy gaps of metalic nanotubes under a local transverse electric field can be used for designing quantum switches[司. Our calculation shows that there exists a critical magnitude of the field strength beyond which the gap starts to decrease and oscillates further on. 
For simplicity, only zigzag (both metallic and semiconducting) and armchair tubes are considered in the full-band TB calculations. The extension of the numerical results to chiral tubes is confirmed by the analytical results within the $\vec{k} \cdot \vec{p}$ method.

\section{Transverse polarizability of a NT in a weak electric field}

An external electrical field creates an induced dipole moment $p$ on the nanotube, which can be estimated through the calculation of the polarizability of $\pi$ electrons [6]. The unscreened linear polarizability $\alpha_{0}(\omega)$ relates the dipole moment $p$ to the total electrical field $\mathcal{E}_{t o t}$ with $p=\alpha_{0} \mathcal{E}_{t o t}$, which accounts for the contribution from the single particle excitation. The dielectric function, defined as $\epsilon=\mathcal{E}_{\text {tot }} / \mathcal{E}_{\text {ex }}$, and the actual (or screened) polarizability $\alpha$ can be easily retrieved from $\alpha_{0}$ as

$$
\epsilon(\omega)=1+2 \frac{\alpha_{0}(\omega)}{R^{2}}, \quad \alpha(\omega)=\frac{\alpha_{0}(\omega)}{\epsilon(\omega)}
$$

As discussed above, when the field strength is less than $t a /\left(e R^{2}\right)$, one can use wavefunctions and energies of the electron states obtained without external perturbation. Results of the TB calculation in the static limit are shown in Figure 1, where metallic and semiconducting tubes are compared. The unscreened polarizability $\alpha_{0}$ is quadratically proportional to $R^{2}$ and is dependent on the conducting properties (symmetry of the tube). As the radius of NTs increases, $\alpha_{0}$ can be fitted by an universal expression:

$$
\alpha_{0}=C R^{2}, \quad C \approx \begin{cases}1.96, & \text { metallic } \\ 2.15, & \text { semiconducting }\end{cases}
$$

The corresponding dielectric function $\epsilon$ is

$$
\epsilon \approx \begin{cases}4.92, & \text { metallic } \\ 5.30, & \text { semiconducting }\end{cases}
$$

which agrees very well with previous results [6, 7]. For comparison, we have calculated the transverse polarizability using the $\vec{k} \cdot \vec{p}$ scheme [8]. This approximation is equivalent to the linerization of the electron energy dispersion in the vicinity of the Fermi points $\pm k_{F}$. The cutoff of $k_{\max }=1 / R$ is used for the calculation and only the lowest subbands are included.

With these approximations, the analytical expression for the polarizability is

$$
\alpha_{0}=C^{\prime} R^{2}, \quad C^{\prime}= \begin{cases}(\sqrt{2}-1) \frac{4 e^{2}}{v_{F} \pi} \approx 1.41, & \text { metallic } \\ \beta \frac{4 e^{2}}{v_{F} \pi} \approx 1.58, & \text { semiconducting }\end{cases}
$$

Here, $v_{F}=\sqrt{3} a t / 2 \approx 5.4 \mathrm{eV} \AA$ is the Fermi velocity, with $\hbar$ taken to be unity. We also have for the integration constant, $\beta \approx 0.463$. The value of $\alpha_{0}$, calculated with the $\vec{k} \cdot \vec{p}$ scheme in Eq. (4)), is smaller than the numerical result obtained from by the full-band calculation, Eq. (2), but it provides a more intuitive understanding of the physical factors which affect the polarizability.

The independence of the dielectric function on the geometry (NT radius) holds only as long as one can verify the condition that the NT bands are half-filled. However, we notice that some nanotubes are naturally doped during the growth process, in which case the Fermi level $E_{F}$ would shift away from the charge neutrality level, and similarly under conditions of charge injection or application of an external bias to the nanotube. In these cases, the polarizability depends on the occupation or depletion of new states.

Let's assume $E_{F}>0$ (note that the sign of $E_{F}$ does not affect the results) and define the unscreened polarizability for a metallic tube as:

$$
\begin{aligned}
\alpha_{0}^{\text {met }}\left(E_{F}, R\right) & =\alpha_{0}^{\text {met }}(0, R)+\Delta \alpha_{0}^{\text {met }}\left(E_{F}, R\right) \\
& =\alpha_{0}^{\text {met }}(0, R)+\Delta \alpha_{0}^{\text {met }, 1}\left(E_{F}, R\right)+\Delta \alpha_{0}^{m e t, 2}\left(E_{F}, R\right)+\cdots
\end{aligned}
$$


where $\Delta \alpha_{0}^{m e t, i}\left(E_{F}, R\right)$ accounts for the contribution due to the occupation of $i$ th conduction subband. At low Fermi energy, the $\vec{k} \cdot \vec{p}$ approximation gives:

$$
\begin{aligned}
& \Delta \alpha_{0}^{m e t, 1}\left(E_{F}, R\right)=\frac{4 e^{2}}{\pi v_{F}^{3}} R^{4} E_{F}^{2} \\
& \Delta \alpha_{0}^{m e t, 2}\left(E_{F}, R\right)=\frac{8 e^{2}}{3 \pi v_{F}^{3}} R^{4} H\left[E_{F}-E_{b 2}\right]\left(E_{F} \sqrt{E_{F}^{2}-E_{b 2}^{2}}-E_{b 2}^{2} \operatorname{arcsinh}\left[\frac{\sqrt{E_{F}^{2}-E_{b 2}^{2}}}{E_{b 2}}\right]\right)
\end{aligned}
$$

Here, $H$ is the unit step function $\left(H[x]=1\right.$ when $x>0$ and 0 otherwise), and $E_{b 2}=v_{F} / R$ is the energy of the bottom of the $2^{\text {nd }}$ subband.

If one considers the low energy properties of nanotubes, the $\vec{k} \cdot \vec{p}$ method provides a reasonable approximation. When the Fermi level is within the first subband, the unscreened polarizability and the dielectric function for a metallic tube of radius $\mathrm{R}$ can be written as:

$$
\begin{gathered}
\alpha_{0}^{m e t}\left(E_{F}, R\right) \approx \alpha_{0}^{m e t}(0, R)+\frac{4 e^{2}}{\pi v_{F}}\left(\frac{E_{F} R}{v_{F}}\right)^{2} R^{2} \\
\epsilon^{m e t}\left(E_{F}, R\right) \approx \epsilon^{m e t}(0)+2 \frac{4 e^{2}}{\pi v_{F}}\left(\frac{E_{F} R}{v_{F}}\right)^{2}
\end{gathered}
$$

where the prefactor $4 e^{2} / \pi v_{F}$ is the dimensionless density of states of the first subband.

As shown above, the shift of Fermi level away from the charge neutrality level changes considerably the polarizability and, consequently, the nanotube dielectric function. The dielectric screening increases with the radius of the tube, according to Eq. (8), in contrast to the screening of a neutral tube, which is independent of the radius. This is because of the strong dependence on $R$ found in the transition energy of the main terms in the expressions for $\epsilon, \Delta E \sim v_{F} / R$. One must conclude that the dielectric properties of a nanotube depend not only on radius and type of bandstructure (metal or semiconductor), but also on the charge carrier density in the tube [9]. This charge density varies with applied field if the tube is connected to electron reservoirs and charge flow through the tube is allowed. Thus, the calculation of the polarizability of the nanotube in a device environment is a complicated problem which has to be solved self-consistently.

\section{$3 \quad$ NT Bandstructure Modification in a strong field limit}

When the applied field is strong enough to mix neighboring subbands, that is $e \mathcal{E} R \geq t a / R$, the bandstructure of a nanotube is considerably modified. We have calculated the energy bands of a $[10,10]$ armchair tube in electrical fields of different strength. Since we are interested in the low energy region of nanotubes, only subbands in the energy range $|E| \leq t$ are shown in Figure 2. We emphasize that the two lowest subbands always cross, even at a very large field, although the bandstructure has been noticeably modified [10]. At $\mathcal{E}=0.1 \mathrm{~V} / \AA$ (see Figure 2(b)), the Fermi points shift toward the $\Gamma$ point $(k=0)$ and the two lowest subbands are flattened near the Fermi points. At the same time, all states which were degenerate with respect to the magnetic number, $\pm m$, split. The splitting becomes more obvious closer to the lowest subbands. The large degeneracy at the first Brillouin zone (FBZ) boundary $k=\pi / a$ is also lifted and bending is observed for all subbands at this point.

As the field strength increases, the two lowest subbands show oscillatory bends with multiple nodes generated, while the first node moves even closer to $k=0$ (Figure 2(c)). For other subbands, the splitting of $\pm m$ subbands become more significant.

This bandstructure modification is clearly seen in the density of states of the nanotube, as shown in Figure 2 $2(d-f)$. As the field is applied, the low energy plateau displays a bump which increases with field. The enhanced DOS near $E=0$ is due to the flattening and bending of the two lowest subbands. On the other hand, the lifting of $\pm m$ degeneracy of all doublets and the bending at the FBZ boundary split the single VHS peaks into multiple ones. Although the DOS structure (Figure 2) changes considerably as compared to the case $\mathcal{E}=0$, all DOS features may be attributed to specific symmetry of the states. We 
stress that many experimental techniques, ranging from Raman scattering to scanning tunneling spectroscopy use high electric fields to probe the electronic properties of a nanotube, which may perturb the underlying electronic structure. Our theoretical results may help to understand disagreements between experimental measurements and predictions for DOS, effective masses and the locations of VHS peaks. For example, we obtained different shifts of optical transition energy $E_{11}$ and $E_{22}$.

For quasi-metallic zigzag tubes, the physics is quite different. At weak fields, a bandgap opens at the Fermi point $k=0$. When the total field is smaller than a critical field $\mathcal{E}_{c}$, the gap is quadratically proportional to the product of the field strength $\mathcal{E}$ and the radius $R$, as shown in Figure 3 . The analytical expression for the gap can be obtained within a degenerate perturbation theory:

$$
E_{g} \sim \frac{(e \mathcal{E} R)^{2}}{6 t}, \mathcal{E}<\mathcal{E}_{c}
$$

When the field increases beyond $\mathcal{E}_{c}$, the minimum gap shifts away from the original Fermi point $k=0$ and the gap starts to decrease. The value of the critical field depends on the nanotube radius (see Figure 3 inset) and is fitted to be:

$$
e \mathcal{E}_{c} R \sim v_{F} / R
$$

Thus, the degenerate perturbation theory which is used in deriving Eq. (9) is no longer valid, when the external potential exceeds the energy distance between neighboring subbands, $\Delta E \propto v_{F} / R$.

It may be interesting for electronics applications to be able to modulate locally the gap of the nanotube. Our study shows that for metallic zigzag NT one can, indeed, open the gap. However the gap cannot exceed some critical value beyond which a further increase of the field begins to close the gap. From Eqs. (9) and (10), the critical bandgap for a metallic zigzag tube [3n, 0$]$ is approximately:

$$
E_{g c} \sim \frac{v_{F}^{2}}{6 t R^{2}}=\frac{\pi^{2}}{2 n^{2}} t
$$

which is almost negligible for nanotubes with large radius $(n>20)$. Although larger radius NTs may seem more attractive due to the smaller critical field is smaller, in these structures the maximum gap will also be smaller. On the other hand, we notice that for very narrow NTs, the $\sigma-\pi$ mixing may results in the opening of secondary gaps, which may prohibit using very narrow NTs for band modulations.

The transverse field effect discussed above, including gap opening or preserved subband crossing as well as degeneracy lifting, can be explained by using methods of group theory. For a detailed description of a group theory technique for nanotubes, we refer the reader to [11]. For armchair and zigzag tubes, the full set of quantum numbers includes the longitudinal momentum $k$, the angular momentum $m$, and parities with respect to the vertical mirror reflection $\sigma_{v}$ and the horizontal mirror reflection $\sigma_{h}$ [11]. Of the $4 n$ bands (for a $[n, n]$ or $[n, 0]$ tube), only those with $m=0, n$ have definite even or odd parity about $\sigma_{v}$ and they are non-degenerate (spin degeneracy is not relevant here and is not discussed). All the other bands are doubly degenerate at zero field with respect to $+m$ and $-m$ indices.

We assume that the components of the electron state on different sub-lattices are not mixed when the magnitude of the field satisfies $e \mathcal{E} a_{c-c} \ll t$, where $a_{c-c}$ is the nearest neighbor distance in the CNT. Thus, a uniform transverse electric field possesses an odd parity with respect to a vertical mirror plane of the nanotube. Due to the commutation of a perturbed Hamiltonian $H=H_{0}+e \mathcal{E} x$ and the vertical parity operator $\sigma_{v}$, parities with respect to $\sigma_{v}$ remain good quantum numbers. For an armchair tube, the two crossing subbands ( $m=n, s= \pm 1$ ) have opposite $\sigma_{v}$ parities.

In the presence of a transverse field, the degeneracy of these two subband at $k_{F}=2 \pi / 3 a$ is lifted in the second order of perturbation theory, but since they have different $\sigma_{v}$ parities, the crossing at the new Fermi points is not prohibited. In contrast, for metallic zigzag tubes, electron states in the four lowest subbands ( $m= \pm 2 n / 3, s= \pm 1$ ) do not have definite $\sigma_{v}$ parities and can be mixed by the external field in a high order perturbation theory by coupling to the states with $m=2 n / 3 \pm 1$. Two pairs of new states with opposite parities with respect to $\sigma_{v}$ are the result. According to the anti-crossing rule for NTs subbands noticed in [12], the degeneracy at $k_{F}=0$ will be lifted. Similarly, the splitting of the degenerate subbands $\pm m$ is explained by their mixing to the other subbands by the transverse field. Because of the selection rule $m^{\prime}=m \pm 1$, the two degenerate states $\left|\psi_{m}^{(0)}\right\rangle$ and $\left|\psi_{-m}^{(0)}\right\rangle$ can only be mixed and split at a high order of perturbation theory. Thus, the coupling strength between $| \pm m\rangle$ states 
depends on $\min [m, n-m]$, which is a "distance" between these two states and the non-degenerate states $|0\rangle$ and $|n\rangle$. This explains why, at low energy, the splitting is more prominent for subbands in armchair tubes, i.e. near $m=n$ and almost indiscernible for subbands in zigzag tubes, i.e. away from $m=0, n$.

In summary, we studied for the first time the effect of doping and/or charge injection on the transverse polarizability of nanotubes. We found that for metallic tubes, the polarizability grows quadratically with $E_{F}$ and scales as $R^{4}$ at low Fermi levels, leading to an enhancement of the dielectric function for the doped nanotubes. With an increase of the the applied field, the bandstructure is considerably modified due to the lowering of symmetry. The zero-gap structure of armchair tubes is always preserved while gap opening and closing occur in metallic and semiconducting zigzag tubes respectively. Degeneracy lifting of $\pm m$ subbands and the flattening of lowest subbands are predicted, which changes the shape and magnitude of the peaks in the density of states.Thus we conclude that formation of multiple valleys in the bandstructure, enhancement of DOS at the Fermi level and engineering of subbands with required effective mass of charge carriers are possible with a transverse electric field. We also predict a maximum bandgap which opens in a metalic tube and give an optimal range of nanotube radius for bandgap engineering.

\section{ACKNOWLEDGMENTS}

This work was partial supported by the National Science Foundation, grant ITR/SY 0121616. One of us (SVR) acknowledges support through a CRI grant of UIUC, DoE grant DE-FG02-01ER45932, NSF grant ECS-0210495 and Beckman Fellowship from the Arnold and Mabel Beckman Foundation

\section{References}

[1] J. Appenzeller, J. Knoch, V. Derycke, et al, Phys.Rev.Lett. 89, 126801 (2002)

[2] P. Kim and C.M. Lieber, Science 286, 2148 (1999); S. Akita, Y. Nakayama, S. Mizooka, et al., Appl. Phys. Lett. 79, 1691 (2001).

[3] M. Dequesnes, S.V. Rotkin and N.R. Aluru, Nanotechnology 13, No 1, 120 (2002).

[4] R. Saito, G. Dresselhaus, M.S. Dresselhaus, Physical Properties of Carbon nanotubes, Imperial College Press (1998)

[5] YH.Kim and K.J.Chang, Phys.Rev.B 64, 153404 (2001)

[6] L.X. Benedict, S.G. Louie, and M.L. Cohen, Phys.Rev.B. 52, 8541 (1995).

[7] D.S. Novikov and L.S. Levitov, cond-mat/0204499 (2002)

[8] T. Ando, T. Nakanishi, and R. Saito, J.Phys.Soc.Jpn. 67, 2857 (1998); P.L. McEuen et al., Phys.Rev.Lett., 83, 5098 (1999).

[9] Our calculation shows that the temperature contribution adds to the dielectric function in Eq. (8) as $\frac{2 \pi^{2}}{3}\left(4 e^{2} / \pi v_{F}\right)\left(k_{B} T R / v_{F}\right)^{2}$. This correction is smaller than $10 \%$ at room temperature for tubes with a moderate radius $(R<32 \AA)$.

[10] This phenomenon is in contradiction to the results of X. Zhou, H. Chen, ZC. Ou-Yang, J.Phys.Cond.Mat. 13, L635 (2001). They found that for a [10,10] nanotube in a transverse field, a gap will open and vary quadratically with the strength of the field.

[11] T. Vuković, I. Milošević, and M. Damnjanović, Phys.Rev.B., 65, 045418 (2002)

[12] M. Damnjanović, T. Vuković, I. Milošević, Solid State Commun. 116, 265 (2000) 


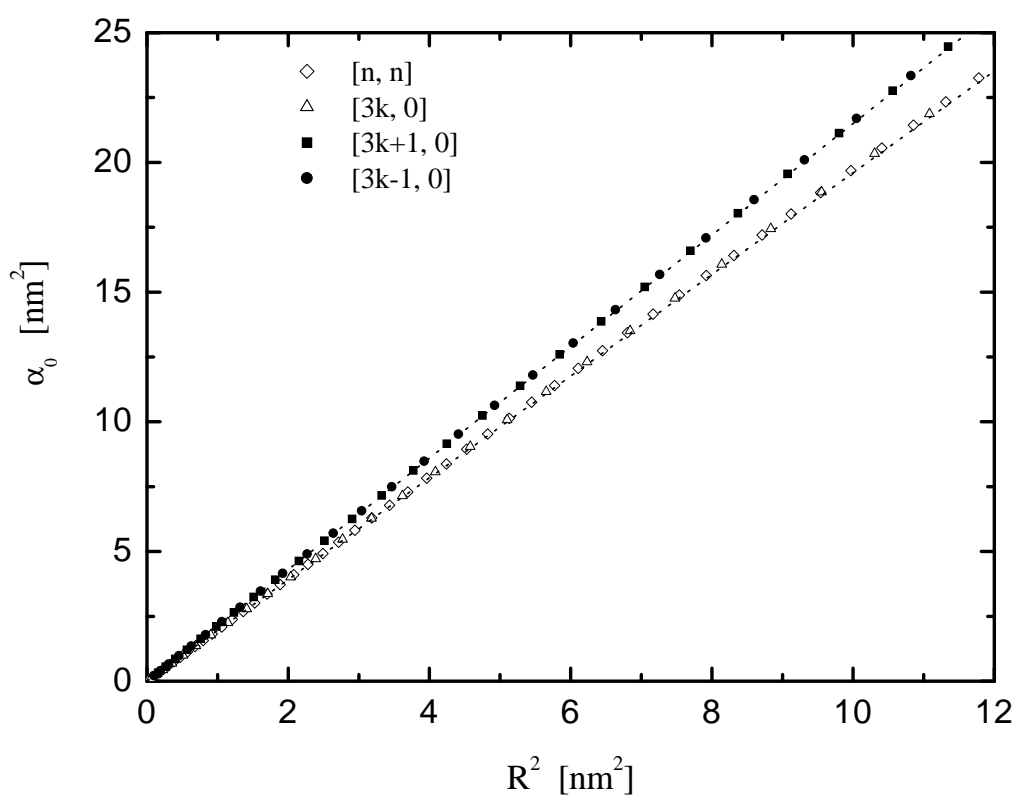

Figure 1: Plot of $\alpha_{0}$ vs. $R^{2}$ for armchair and zigzag tubes. The fitting lines are $\alpha_{0}=1.96 R^{2}$ and $\alpha_{0}=2.15 R^{2}$ respectively . 

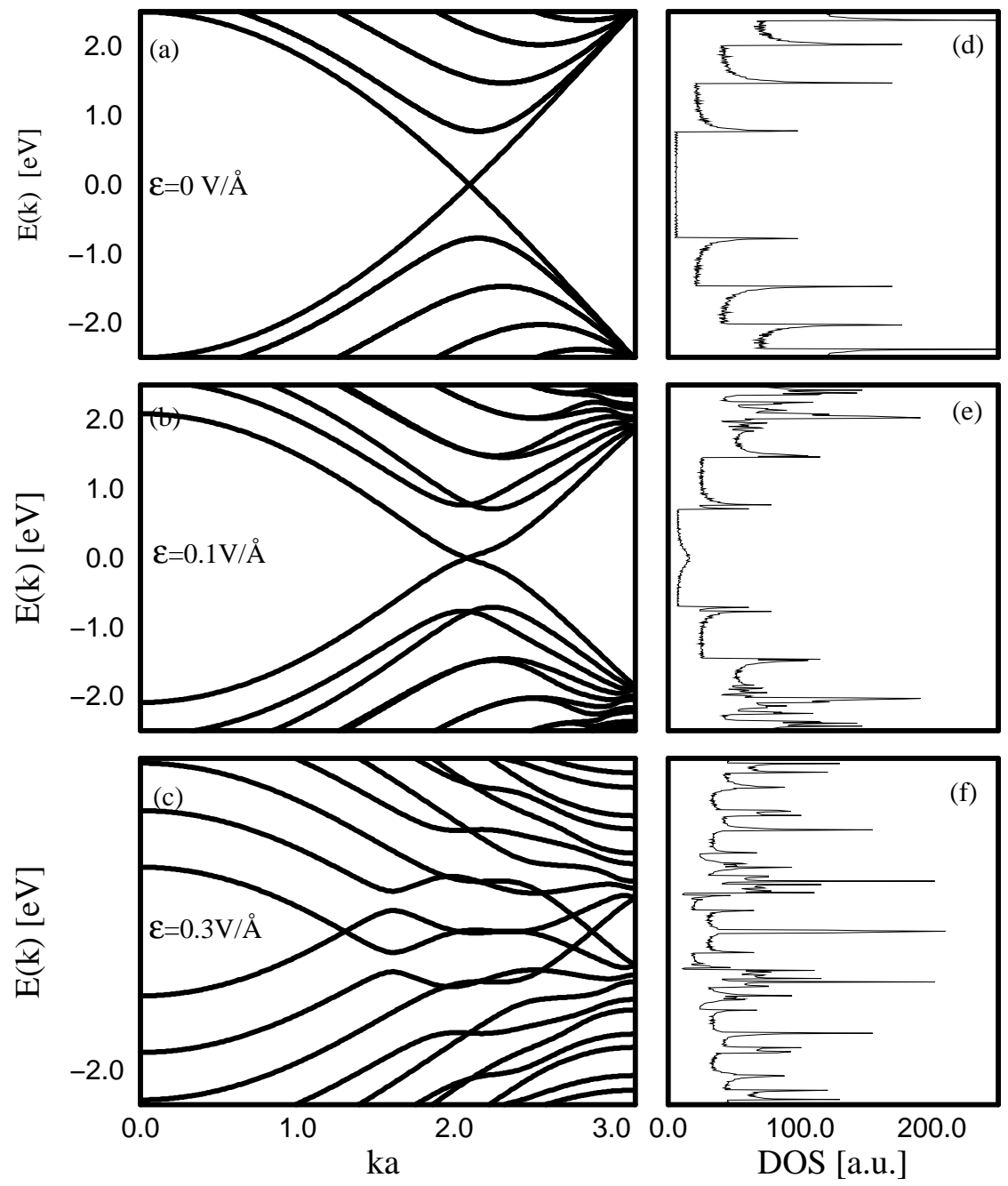

Figure 2: Band structures ((a),(b),(c)) and DOS ((d),(e),(f)) of a $[10,10]$ armchair tube at various transverse electric fields: $\mathcal{E}=0,0.1,0.3 \mathrm{~V} / \AA$. Higher bands $(E>2.5 \mathrm{eV})$ are not displayed. 


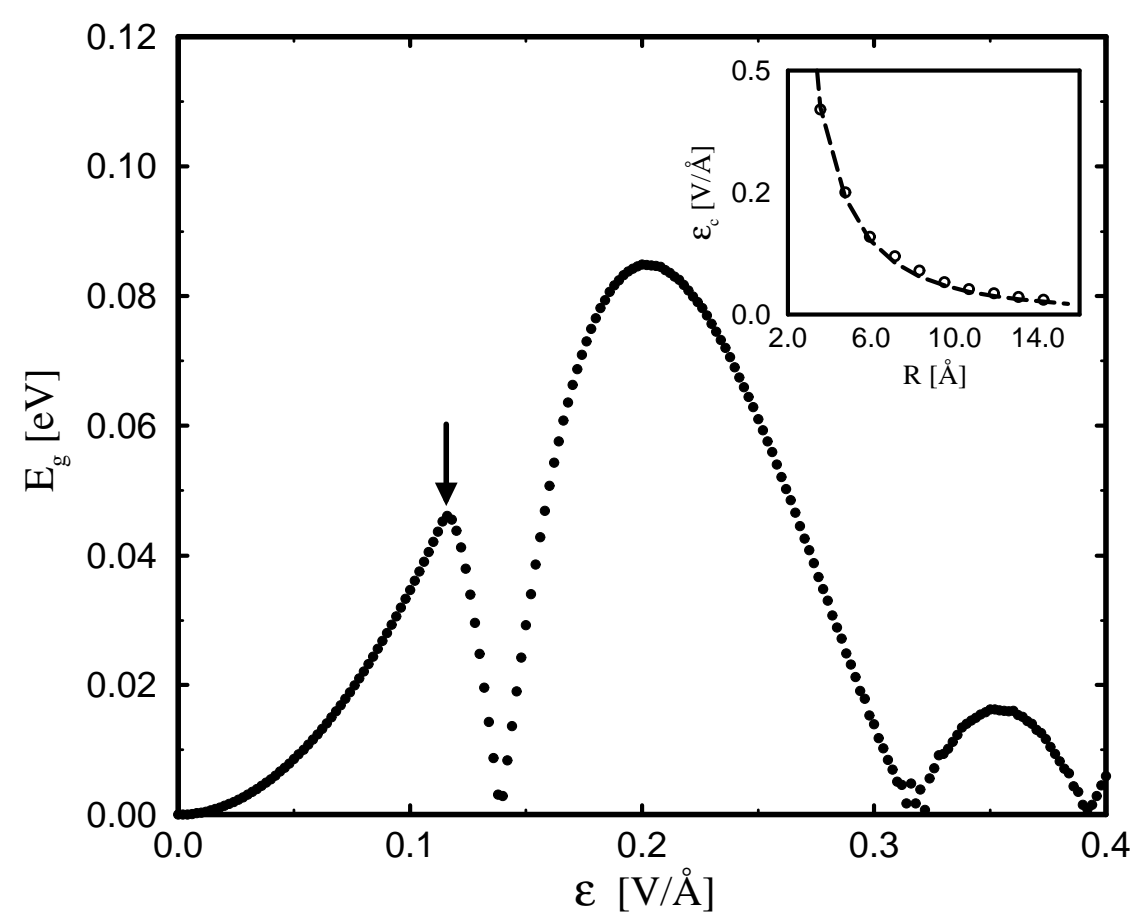

Figure 3: Bandgap variation of a $[18,0]$ tube with increasing field strength. The arrow indicates the critical field strength $\mathcal{E}_{c}$ (see text). Inset: $\mathcal{E}_{c}$ as a function of the radius $R$. Open circles represent the numerical results for metallic zigzag tubes and the dashed line is the fitting curve $\mathcal{E}_{c}=v_{F} / e R^{2}$. 